\title{
Image Analysis Techniques on Phenotype for Plant System
}

\author{
Karthiga Rani D.
}

\begin{abstract}
In recent years, there is a rapid advancement in computer vision technology which is much effective in extracting useful information from plant images in the field of plant phenomics. Phenomic approaches are widely used in the identification of relationship between phenotypic traits and genetic diversities among the plant species. The need for automation and precision in phenotyping have been accelerated by the significant advancement in genotyping. Regardless of its significance, the shortage of freely available research databases having plant imageries has significantly obstructed the plant image analysis advancement. There were several existing computer vision techniques employed in the analysis of plant phenotypes. Conversely, recent trends in image analysis with the use of machine learning and deep learning based approaches including convolutional neural networks have increased their expansion for providing high-efficiency phenotyping of plant species. Thus, to enhance the efficiency of phenotype analysis, various existing machine learning and deep learning algorithms have been reviewed in this paper along with their methods, advantages, and limitations.
\end{abstract}

Keywords : Phenomics, machine learning, deep learning, phenotyping, convolutional neural networks.

\section{INTRODUCTION}

$\mathrm{P}_{\text {lant phenotyping is the analysis of the changes in the }}$ performance and appearance of plants caused due to genotypic variations and the surrounding environments in which the plants are exposed. Wide research in plant phenotyping is a crucial factor to meet the agricultural demands in the future and in enabling the less usage of land, water and fertilizer. Image analysis is developing as an important technique in plant phenotyping. These phenotypic approaches are useful in the identification of interactions prevailing in-between phenotypic traits and genetic diversities in plants. This could also be accomplished by analyzing the non-invasive quantitative parameters which reflect physiological states and traits all through the life of plants. The requirement for automation and precision in phenotyping have been accelerated by the significant advancement in genotyping. The phenotyping rate is not found to be match with the growing genotyping rate and thereby developing a bottleneck. The integration of effective DNA sequencing platforms and plant phenotyping afford opportunities for the exploration of genetic factors such as environmental tolerance, growth, disease resistance and production. The mapping of genotype to phenotype ratio with

Revised Manuscript Received on December 16, 2019.

* Correspondence Author

Karthiga Rani *, Department of Computer Science and IT, N.M.S Sermathai Vasan College for Women, Madurai, India. Email: karthigakumaresh@yahoo.in the use of statistical methods like GWASs (genome-wide association Studies). Machine learning have been employed in different applications that includes image analysis. The steps typically followed in image analysis are represented as follows: preprocessing or data augmentation, segmentation of the preprocessed data, feature extraction followed by classification. Algorithm on the basis of deep learning depicts most accurate results when comparing with conventional techniques like plantCLEF. Further machine learning based algorithm offer distinct features that helps in the dissection of complex traits and in the determination of visual signatures linked with the plant traits. The algorithm implied in plant phenotyping must be combined with reliable platforms and should process with plenty of data developed in the experiments. So that this review focus on the development of robust, accurate and an automated analytical algorithm for image analysis for the improvement in crop yield with respect to climate change and population demography.

This survey is structured as follows. Section II offers various state-of-art preprocessing techniques involved in the image analysis of plant phenotype. Section III explains various segmentation algorithms for image analysis of plant phenotype. Section IV provides the overview of Traditional feature extraction techniques employed for plant phenotyping. Section $\mathrm{V}$ provides the detailed depiction of classification approaches in plant phenomics. Numerous datasets used in existing approaches are described in section VI. Finally, section VII concludes this study.

\section{DIFFERENT PREPROCESSING APPROACHES IN PLANT PHENOTYPING}

K.mochida,et al.,(2018) said about Preprocessing is the initial step in image processing which is involved in the data organization that aids the succeeding steps to derive effective results. In particular, when field images under uncontrolled environment are employed, this preprocessing technique enhances the quality of image processing. Data transformation methods like conversion of gray scale, cropping steps, standardization and contrast improvement are implemented in preprocessing. To generalize the pattern analysis, variation in dataset images are increased by means of data augmentation. Scaling of images, addition of noise and flipping are the methods that are used frequently implied in data augmentation. This process increases the dataset size and reduces over fitting.[1] 
K.Keller,el al.,(2018) was achieved validation and training by splitting 285 labelled images into 45 validation and 20 training pairs. It is followed by three steps in which each image was made to undergo a flip a mirror, and a ninety degree rotation so as to yield final dataset. This suggested work employed both augmented and original dataset correspondingly in order to test the efficiency of the augmentation. Preprocessing have been recognized as a reliable level for effective segmentation[2]. H.TAttari and A.Ghafari-Beranghar(2018) implemented an algorithm to reduce the noise of the plant thereby improve the segmentation process. The noise had been reduced by color space conversion and binarization methods. At last this work compared the suggested algorithm with the existing algorithms.[3]

\section{VARIOUS SEGMENTATION ALGORITHMS FOR IMAGE ANALYSIS OF PLANT PHENOTYPE}

This section provides the detailed survey on various existing segmentation approaches employed for the image analysis on plant phenotypes.

Generally, segmentation represents the most significant and initial step. It is employed for the extraction of target information from the image data that is preprocessed by separating pixel sets in the image. It also helps in recognition and quantification of specific plant organs automatically.

H.Lu,,et al.,(2017)explained a work on maize tassels in the wild with the use of local count regression networking approach. It was essential to count the tassels of maize accurately so as to monitor the progress of maize plant growth. This work have done usually with the aid of man power and it was regarded as the most tedious task. In the modern plant phenotyping context, automation process was the most essential one for facing a huge scale analysis of plant phenotype and genotype. Due to the emergence of increased computational resources and large scale datasets, a computer vision technology turn out to be the most substantial innovation in recent years. Image based approaches were gaining more courtesy in the studies related to plant system. In order to count the maize tassels automatically, deep convolutional neural network and Tassel Net have been applied in this work for an effective segmentation process. However, this work proves its superiority it also have some drawbacks like reduced rate of counting performance.[4]

H.Scharr,et al.,(2016) described the segmentation of leaves in the plant phenotype analysis. The segmentation of each and every leaves in the specific image was the major contributing task in this work. Consider a usual rosette model plant Arabidopsis and the young tobacco. Even though, the leaves share their characteristics of shape and appearance, there were some challenges like:

- The availability of occlusions and variability at leaf shape and pose,

- The imaging conditions

The major intention of this paper was to compare several solutions employed for leaf segmentation thereby conducting some typical phenotypic experiments. For the plant segmentation, a simple color-based approach has beenemployed from which the results were refined with the use of raster graphics editing software tool. A segmentation process using 3D histogram could be carried through IPK pipeline approach which in turn employs distance amp and unsupervised clustering for segmentation. Also, watershed algorithm was applied to attain the individual segmentation of leaves. Moreover, some approaches were still in lack of individual leaf segmentation.[5]

J.M.Pape and C.Klukas(2015) stated the difficulty of segmentation of individual leaves in the plant images. The accurate identification of the areas of individual leaves helps in enhancing the evaluation of biomass and plant performance. In plants, the counting of leaves was also the most substantial one for recognizing the progress of plant stages. A novel method has been developed for the detection of particular borders of leaf and to resolve incorrect segmentation of leaf due to leaf overlapping.[6]

P.Sodhi et al.,(2017) described the approach of in-field segmentation and the recognition of plant structures with the use of 3D image. An automated approach for mapping the 3D images in the outdoor field was presented for the process of segmentation. The segmentation techniques are then evaluated and were analyzed effectively for both greenhouse and in-field environment. [7]

These existing techniques attempts for proving the segmentation accuracy thereby enabling the identification and quantification of plant organs automatically and estimating the yields of fruits and grains and biomass. This work also aims at enhancing the reproducibility in plant phenotype by means of swapping traditional human-dependent approach of phenotyping, every so often which was a labor demanding and time consuming aspect.

\section{TRADITIONAL FEATURE EXTRACTION TECHNIQUES FOR PLANT PHENOTYPING}

Feature extraction is a process of developing accurate and reliable data that is used for the representation of the image satisfactorily. Since the efficiency of the pattern analysis depends greatly on the feature quality, various approaches are under investigation for phenotyping.

The features are selected on the basis of object characteristics of images like gradient texture, pixel intensity and shape. K.Sabanci,et al.,(2017) had choosed the features like color, shape, homogeneity, correlation and entropy from wheat for the classification in his work. Of the various approaches involved, CNN have been proved to be very effective which is involved in the automatic extraction and classification of the features from the images. Previous research showed feature extraction on the basis of CNN advantages in plant [8] phenotyping. It enables the learning of hierarchical features by means of taxonomical categorization on leaf datasets. [6] The feature extraction in this paper provided the data regarding the leaf shape, leaf overlapping and leaf border. The study detected the issues like less sensitivity in case of small leaves. Machine learning associated with IAP software have been employed that used more number of phenotypic features. These outcomes are implied in the modification of leaf sensitivity in the suggested leaf labelling techniques. [9] described an classification approach for the plant phenotype using elliptical Fourier descriptor also the texture feature set named Haralick's 
texture descriptors for characterizing the plant seed for classification based on taxonomy. Using typical tool for the process of feature extraction, Scale Invariant Features Transforms (SIFT) was employed. This in turn performs as an invariant feature descriptor in terms of rotation, illumination, and viewpoint despite the scaling factor.

\section{STATE-OF-ART CLASSIFICATION APPROACHES IN PLANT PHENOTYPE}

This section offers an overview of several existing approaches that were employed in the process of plant phenotype classification.

The outcomes of preprocessing, segmentation, and feature extraction, were attained in the classification stage. There were several existing machine learning based classification approaches. These ML based approaches were applied hugely in phenotypes of plant recently. The classification techniques in turn highlights two main applications like classification based on taxonomy and physiological state of plant classification.

[ $\underline{8}$ ] introduced a computer-vision dependent methodology for the classification of wheat grains with the use of artificial neural networks. A novel artificial neural network based on the multi-layer perceptron approach was employed for the perfect classification of the wheat grains into the durum or bread. The images were taken by camera of high resolution and are preprocessed initially. Then, the feature extraction process was carried out for the extraction of visual features like three color features and five texture. These visual feature datasets were regarded as the classifier's (ANN) input. The classifier model was trained using 180 grains and testing the accuracy level with 20 grains out of 200 wheat grains. These technique could integrated with industry easily for classifying the agricultural grains.

K.Sabanci,et al.,(2017) presented a computer-vision classification approach for grains with the use of adaptive Neuro-fuzzy inference system. About 200 wheat grain Images taken from high resolution camera were considered as input. The features are extracted on the basis of color (\#3), texture (\#5), and dimension (\#4) and were carried as an input for the classifier. The categorization of subset features were made for evaluating the classification effects.[10]

E.C.Oerke,et al.,(2016) employed Classification techniques that were based on computer-vision technology widely for the recognition of disease symptoms in plants. A technique of hyper spectral imaging was useful in the detection of downy mildew signs occurred in grapevine plants by Plasmopara viticola [11]. Nowadays, deep-learning dependent approaches leads to some enhancement in the accuracy and throughput in the plant disease symptoms analysis.

S.P.Mohanty,et al.,(2016) revealed the probability of applying a deep CNN approach for the identification of 26 diseases appeared in 14 crops. The popular fine-tuned pre-trained deep $\mathrm{CNN}$ architectures were applied, like Google Net and Alex Net[12]. A.Krihevsky widely presented dataset containing 54,306-images of unhealthy and healthy plants from the Plant Village[13].A.C.Cruz,et al.,(2016) presented in his paper a Transfer learning was also utilized for training $\mathrm{CNN}$ models so as to identify the crop disease symptoms like olives [14].

\section{DATASETS EMPLOYED IN EXISTING PLANT PHENOTYPING ANALYSIS}

Different datasets for phenotyping are available for the development of analytical techniques. A peculiar dataset is presented in Arabidopsis that can be used as a fundamental dataset for the development and growth of plant model in computer vision-based plant phenotyping. Further a plant CV website presented datasets for grass species. The significance of phenotypes, traits and gene functions could enable data integration from phenomics and genomics. M.Minervini,et al.,(2019) used standard datasets of annotated and raw rosette images [15]. The material of the plant, and image setup were also described in his paper that are accessible at "http://www.plant-phenotyping.org/datasets"[16], a mobile app. Plantix that implies deep learning and crowd sourced database facilitates customized preferences and diagnosis for the identification of plant diseases, deficiencies and pests on different crops worldwide.

Table 1 A Study on different existing approaches

\begin{tabular}{|c|c|c|c|c|}
\hline Author & Technique & Methodology & Algorithm used & $\begin{array}{l}\text { Advantage/ } \\
\text { disadvantage }\end{array}$ \\
\hline $\begin{array}{l}\text { M.Henke,et } \\
\text { al.,(2019)[17] }\end{array}$ & Preprocessing & $\begin{array}{l}\text { A preprocessing algorithm was presented to } \\
\text { eliminate the noise and separate foreground } \\
\text { from the } 16 \text { background which results the } \\
\text { plant image to help the plant image } \\
\text { segmentation. The preprocessing } 17 \text { is one of } \\
\text { important levels has effect on better image } \\
\text { segmentation and finally better plant's image } \\
18 \text { labeling and analysis. Our proposed } \\
\text { algorithm is focused on removing noise such } \\
\text { as converting the } 19 \text { color space, applying the } \\
\text { filters and local adaptive binarization step such } \\
\text { as Niblack's. }\end{array}$ & $\begin{array}{l}\text { Niblack's } \\
\text { binarization }\end{array}$ & $\begin{array}{l}\text { Niblack's method is } \\
\text { more effective than } \\
\text { the } 298 \text { other } \\
\text { binarization such as } \\
\text { Otsu, Sauvola, and } \\
\text { Bernsen }\end{array}$ \\
\hline $\begin{array}{l}\text { S.T.Namin,et } \\
\text { al.,(2018)[18] }\end{array}$ & Feature extraction & $\begin{array}{l}\text { Deep phenotyping: deep learning for temporal } \\
\text { phenotype/genotype classification }\end{array}$ & $\begin{array}{l}\text { Fourier } \\
\text { descriptor, and } \\
\text { GLCM based } \\
\text { feature extraction }\end{array}$ & $\begin{array}{l}\text { Reduced rate of } \\
\text { robustness }\end{array}$ \\
\hline
\end{tabular}




\begin{tabular}{|c|c|c|c|c|}
\hline $\begin{array}{l}\text { J.M.Pape and } \\
\text { C.Klukas, (2015) } \\
{[6] .}\end{array}$ & $\begin{array}{l}\text { Segmentation/Feature extraction/ } \\
\text { classification }\end{array}$ & $\begin{array}{l}\text { Utilizing machine learning approaches to } \\
\text { improve the prediction of leaf counts and } \\
\text { individual leaf segmentation of rosette plant } \\
\text { images }\end{array}$ & $\begin{array}{l}\text { Leaf-Count } \\
\text { Regression } \\
\text { Model }\end{array}$ & $\begin{array}{l}\text { Better Prediction of } \\
\text { leaf counts and to } \\
\text { segment individual } \\
\text { leaves }\end{array}$ \\
\hline $\begin{array}{l}\text { D.Vukadinovic } \\
\text { and G.Polder } \\
(2015)[19]\end{array}$ & Segmentation/classification & $\begin{array}{l}\text { Watershed and supervised classification based } \\
\text { fully automated method for separate leaf } \\
\text { segmentation. Pixel classification based plant } \\
\text { segmentation and watershed based separate } \\
\text { leaves segmentation. }\end{array}$ & $\begin{array}{l}\text { Watershed } \\
\text { algorithm }\end{array}$ & highest accuracy \\
\hline $\begin{array}{l}\text { A.kayabasi,et } \\
\text { al.,(2018) [20] }\end{array}$ & Classification & $\begin{array}{l}\text { An application of ANN model trained by } \\
\text { artificial bee colony (ABC) optimization } \\
\text { algorithm was presented for classification of } \\
\text { the wheat grains into bread and durum. ABC } \\
\text { algorithm is used to optimize the weights and } \\
\text { biases of three-layer multilayer } \\
\text { perceptron(MLP)based ANN }\end{array}$ & $\begin{array}{l}\text { ANN Trained by } \\
\text { ABC Algorithm }\end{array}$ & $\begin{array}{l}\text { It can achieve } \\
\text { accuracy rate of about } \\
100 \%\end{array}$ \\
\hline $\begin{array}{l}\text { T.M.Giselsson,et } \\
\text { al.,(2017)[21] }\end{array}$ & Classification & $\begin{array}{l}\text { A database of images of approximately } \\
960 \text { unique plants belonging to } 12 \text { species at } \\
\text { several growth stages is made publicly } \\
\text { available. It comprises annotated RGB images } \\
\text { with a physical resolution of roughly } 10 \text { pixels } \\
\text { per mm. }\end{array}$ & $\begin{array}{ll}\text { naive } & \text { Bayes } \\
\text { classifier } & \end{array}$ & $\begin{array}{l}\text { It is capable of } \\
\text { maintain constant } \\
\text { result } \\
\text { Disadvantage: it is } \\
\text { sensitive to correlated } \\
\text { attributes. }\end{array}$ \\
\hline
\end{tabular}

11. E.-C. Oerke, et al., "Hyperspectral phenotyping of the reaction of grapevine genotypes to Plasmopara viticola," Journal of experimental botany, vol. 67, pp. 5529-5543, 2016.

\section{CONCLUSION}

This survey provides the overview of various existing techniques implemented for the analysis of plant phenotypes. Computer vision based technology in plant phenotyping is a fast developing field that combines plant science knowledge, machine learning, mechanical engineering and spectral sensing. Highly efficient image analysis of plant phenotyping enable the experiments to perform with different genetic resources. Further recent advances in machine learning approaches plays vital roles in the forecasting of traits by means of genotypic and phenotypic relationship. Progression in CNN based algorithm and plant image dataset for plant phenotyping achieved significant improvement in image analysis and taxonomic classification. This review focused the implementation of efficient, accurate and an automated analytical algorithm for image analysis.

\section{REFERENCES}

1. K. Mochida, et al., "Computer vision-based phenotyping for improvement of plant productivity: a machine learning perspective," GigaScience, vol. 8, p. giy153, 2018.

2. K. Keller, et al., "Soybean Leaf Coverage Estimation with Machine Learning and Thresholding Algorithms for Field Phenotyping," Proceedings of BMVC 2018, p. 0032, 2018.

3. H. Attari and A. Ghafari-Beranghar, "An Efficient Preprocessing Algorithm for Image-based Plant Phenotyping," 2018.

4. H. Lu, et al., "TasselNet: counting maize tassels in the wild via local counts regression network," Plant methods, vol. 13, p. 79, 2017.

5. H. Scharr, et al., "Leaf segmentation in plant phenotyping: a collation study," Machine vision and applications, vol. 27, pp. 585-606, 2016.

6. J.-M. Pape and C. Klukas, "Utilizing machine learning approaches to improve the prediction of leaf counts and individual leaf segmentation of rosette plant images," Proceedings of the Computer Vision Problems in Plant Phenotyping (CVPPP), pp. 1-12, 2015.

7. P. Sodhi, et al., "In-field segmentation and identification of plant structures using 3D imaging," in 2017 IEEE/RSJ International Conference on Intelligent Robots and Systems (IROS), 2017, pp. 5180-5187.

8. K. Sabanci, et al., "Computer vision-based method for classification of wheat grains using artificial neural network," Journal of the Science of Food and Agriculture, vol. 97, pp. 2588-2593, 2017.

9. M. Lo Bianco, et al., "Morpho-colorimetric characterisation of Malva alliance taxa by seed image analysis," Plant Biology, vol. 19, pp. 90-98, 2017.

10. K. Sabanci, et al., "Grain classifier with computer vision using adaptive neuro-fuzzy inference system," Journal of the Science of Food and Agriculture, vol. 97, pp. 3994-4000, 2017.
12. S. P. Mohanty, et al., "Using deep learning for image-based plant disease detection," Frontiers in plant science, vol. 7, p. 1419, 2016.

13. A. Krizhevsky, et al., "Imagenet classification with deep convolutional neural networks," in Advances in neural information processing systems, 2012, pp. 1097-1105.

14. A. C. Cruz, et al., "X-FIDO: An effective application for detecting olive quick decline syndrome with deep learning and data fusion," Frontiers in plant science, vol. 8, p. 1741, 2017

15. M. Minervini, et al., "Finely-grained annotated datasets for image-based plant phenotyping," Pattern recognition letters, vol. 81, pp. 80-89, 2016.

16. "http://www.plant-phenotyping.org/datasets."

17. M. Henke, et al., "Comparison and extension of three methods for automated registration of multimodal plant images," Plant methods, vol. 15, p. 44, 2019.

18. S. T. Namin, et al., "Deep phenotyping: deep learning for temporal phenotype/genotype classification," Plant methods, vol. 14, p. 66 2018.

19. D. Vukadinovic and G. Polder, "Watershed and supervised classification based fully automated method for separate leaf segmentation," in The Netherland Congress on Computer Vision, 2015, pp. $1-2$

20. A. Kayabasi, "An Application of ANN Trained by ABC Algorithm for Classification of Wheat Grains," International Journal of Intelligent Systems and Applications in Engineering, vol. 6, pp. 85-91, 2018.

21. T. M. Giselsson, et al., "A public image database for benchmark of plant seedling classification algorithms," arXiv preprint arXiv:1711.05458, 2017

\section{AUTHORS PROFILE}

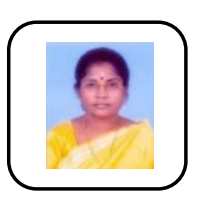

D.Karthiga Rani Currently working as Assistant Professor, Department of Computer Science in N.M.S.Sermathai Vasan of college for women „Madurai,Tamil Nadu,India since 1995,. She obtained Masters Degree in Computer Science from N.M.S.Vellaichamy Nadar College, Madurai under Madurai kamaraj universityi, M.Phil Degree in Computer Science in mother Teresa universityand now purusing PhD. His area of interests includes, Image Processing, Artificial Intelligence, Data Mining and Networks. She has presented more than 5 papers in National and International conferences. She guided 3 Mphil scholars. 